\title{
Dermatology
}

Dermatology 2009;218:186-187

DOI: $10.1159 / 000163083$

\section{Filaggrin Mutation c.3321delA in a Korean Patient with Ichthyosis Vulgaris and Atopic Dermatitis}

Tae-Won Kang, Jeong Seon Lee, Se-Woong Oh, Soo-Chan Kim Department of Dermatology and Cutaneous Biology Research Institute, Yonsei University College of Medicine, Seoul, Korea

\section{Key Words}

Filaggrin mutation $\cdot$ Ichthyosis vulgaris $\cdot$ Atopic dermatitis

Filaggrin plays a major role in the production of the epidermal barrier function. It is initially produced as profilaggrin in the granular layer and is then processed to filaggrin at the junction of the granular layer and corneal layer of the epidermis. Filaggrin functions to aggregate keratin filaments so that effective corneocytes are produced. Filaggrin is then degraded into individual amino acids, becoming the natural moisturizing factor of the stratum corneum. Filaggrin deficiency has obvious adverse effects on stratum corneum function.

Atopic dermatitis (AD) and ichthyosis vulgaris (IV) are relatively common heritable skin diseases. Recent studies showed that both diseases are closely related to loss-of-function mutations in the filaggrin gene (FLG). The homozygous or compound heterozygous loss-of-function mutations p.R501X and c.2282del4 in FLG have been identified in moderate or severe IV patients from Ireland, Scotland and the USA [1]. These variants are carried by approximately $9 \%$ of European people and were also revealed to be important predisposing factors for $\mathrm{AD}$ [2]. Meanwhile, c.3321delA and p.S2554X mutations in FLG were identified as prevalent in Japanese IV and AD patients [3]. However, in that study the frequency of FLG mutations in the Japanese AD cohort was much lower than that seen in Europeans (5.6 and 48\%, respectively) [3]. Recently, the same research group has reported additional nonsense FLG mutations (p.S2889X and p.S3296X) in Japan and extended the statistical significance of FLG null mutations in Japanese cases to $>20 \%$ [4]. These findings imply that FLG mutations are predisposing factors for IV and $\mathrm{AD}$, and show the presence of population-specific recurrent FLG mutations.

In this regard, we analyzed FLG mutations in a 35 -year-old Korean man who presented with a history of IV and AD since childhood. His sister and 2 daughters were affected by AD and xerosis. Marked adherent fine scales were clearly visible on his trunk. He also showed hyperlinear palms (fig. la, b). Histology revealed no keratohyalin granules in the granular layer (fig. 2). We performed direct sequencing of the FLG gene in this patient, as well as screening for the previously reported mutations. Analysis revealed that he was a heterozygous carrier of the FLG mutation c.3321delA (fig. 3). This mutation has already been reported by Nomura et al. [3] in Japanese cases.
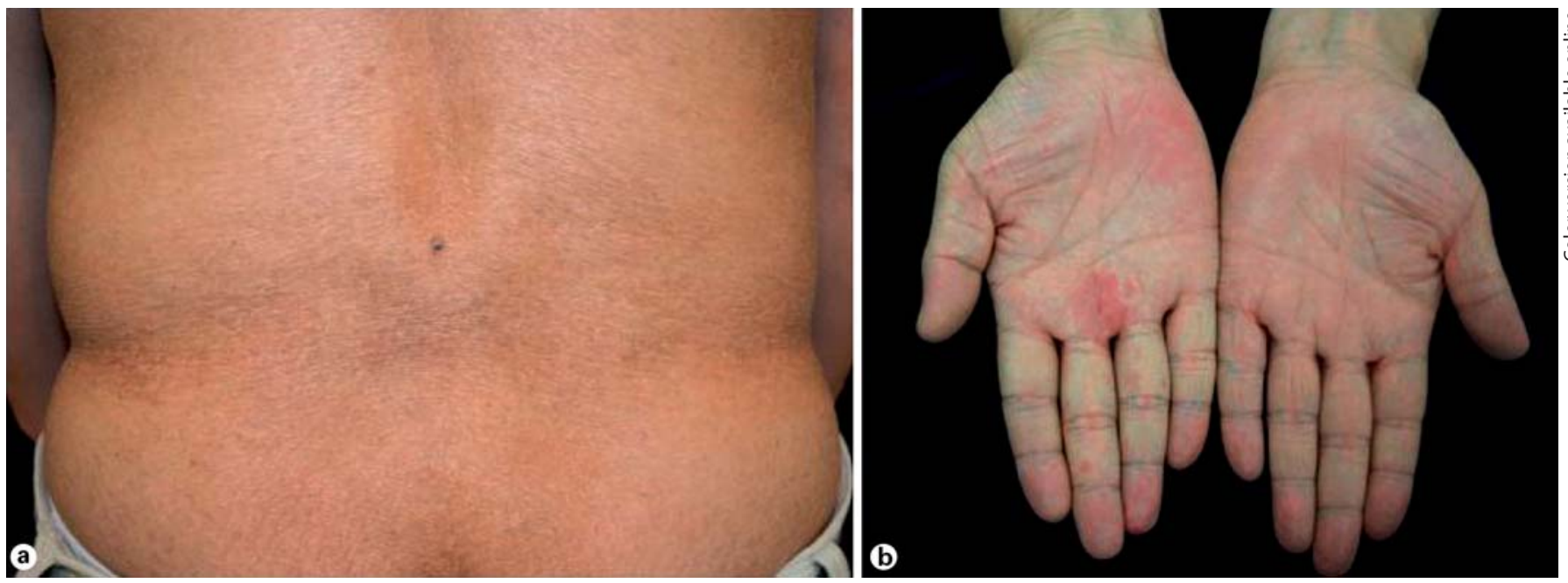

Fig. 1. Clinical features of the patient with IV and AD, showing marked adherent fine scales on the trunk (a) and hyperlinear palms (b). 


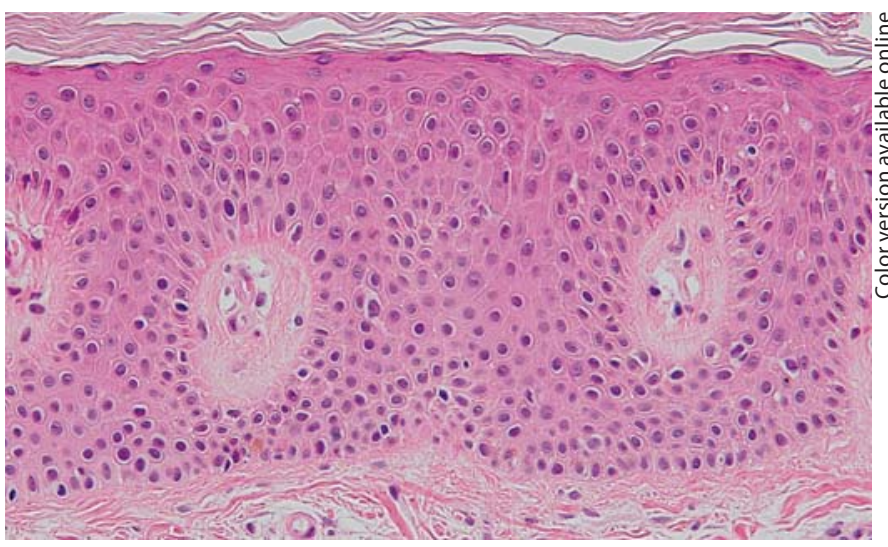

Fig. 2. No keratohyalin granules are seen in the upper granular layer. HE. $\times 400$.

From this finding, we can assume that at least some proportion of $\mathrm{AD}$ and/or IV patients of Korean origin are affected by FLG null mutations, and that population-specific recurrent mutations are closely related to Japanese cases. Further extensive casecontrol studies are required to reveal the contributing effects of FLG mutations in both diseases in the Korean population. Also, further FLG mutation analysis in different populations will uncover the full spectrum of mutations and assess their contributions to both diseases globally.

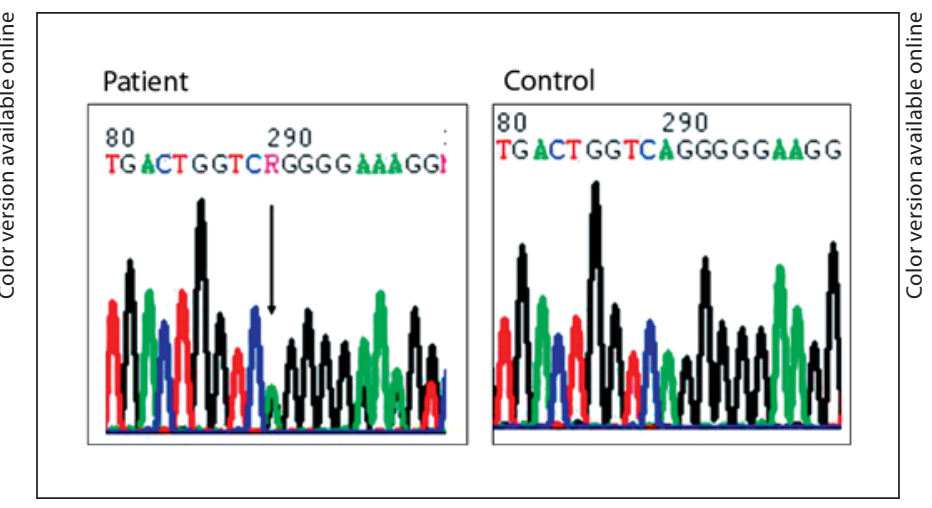

Fig. 3. Detection of FLG mutation c.3321delA. A heterozygous deletion mutation in filaggrin repeat 2 in exon 3, 3321delA, was identified in the patient.

\section{References}

1 Smith FJD, Irvine AD, Terron-Kwiatkowski A, et al: Loss-of-function mutations in the gene encoding filaggrin cause ichthyosis vulgaris. Nat Genet 2006;38:337-342.

-2 Palmer CNA, Irvine AD, Terron-Kwiatkowski A, et al: Common lossof-function variants of the epidermal barrier protein filaggrin are a major predisposing factor for atopic dermatitis. Nat Genet 2006;38: 441-446.

3 Nomura T, Sandilands A, Akiyama M, et al: Unique mutations in the filaggrin gene in Japanese patients with ichthyosis vulgaris and atopic dermatitis. J Allergy Clin Immunol 2007;119:434-440.

4 Nomura T, Akiyama M, Sandilands A, et al: Specific filaggrin mutations cause ichthyosis vulgaris and are significantly associated with atopic dermatitis in Japan. J Invest Dermatol 2008;128:1436-1441.

\section{Soo-Chan Kim, MD, PhD}

Department of Dermatology, Yonsei University College of Medicine Yongdong Severance Hospital, 146-92, Dogok-dong, Kangnam-gu Seoul 135-720 (Korea)

Tel. +82 22019 3360, Fax +82 23463 6136, E-Mail kimsc@yuhs.ac 